\title{
La geografía itinerante de la cultura
}

\section{The itinerant geography of culture}

\author{
GonzÁlez, Felipe (2012), Megalópoli Y CULTURA. DeL RITUAL INDÍGENA \\ aL PERFormanCE URBANO, Universidad Autónoma del Estado de \\ MÉXICO-PorrúA, MÉXICO, 249 PP., ISBN: 978-607-40 I-550-8.
}

No hay duda que el ámbito de análisis de la cultura es cada vez más complejo y que en consecuencia resulta indispensable explorar visiones que trasciendan los panoramas tradicionales y que aborden la complejidad de las adscripciones identitarias y los umbrales en que los actores de la sociedad van definiendo sus interacciones, sus metas y sus diagnósticos de lo cotidiano.

En este sentido, el trabajo que ha realizado Felipe González Ortiz desde hace varios años, se ha enfocado en hacer evidente la relación entre el desarrollo metropolitano y la cultura, obligando a los lectores de sus textos a polemizar desde diferentes posiciones intelectuales. En el documento que se reseña a continuación, la discusión queda sembrada en el público especializado y hace necesario ser comentada también entre aquellos que buscan una descripción itinerante de las tradiciones en contextos de cambio social y que pretenden conocer e imaginar la realidad circundante desde una perspectiva simbólica.

Esta reseña hace un reconocimiento a las aportaciones del autor, sin embargo, también expresa diferencias analíticas en algunos conceptos y enfoques, reiterando la valía metodológica del texto, aunque también resalta diferentes posibilidades de interpretación, así como la necesidad de precisar información relevante, reconociendo el esfuerzo que expresa el trabajo de campo y los resultados de la investigación.

No hay duda que el documento invita a la reflexión, a la crítica, al análisis y a la discusión, tal y como se presenta a continuación en las principales líneas temáticas que componen el contenido del libro, espero que esta estrategia descriptiva le haga justicia a la ruptura de las descripciones lineales en los mismos términos que busca hacerlo Felipe González.

Una aportación del libro Megalópoli y cultura es el esfuerzo por mostrarnos la diversidad de asimilación y reproducción de las expresiones culturales a través de los saberes, conocimientos y prácticas performativas que le dan congruencia a las acciones sociales. Coincido plenamente con el autor cuando considera que la cultura no es un concepto unitario y se puede explicar como la formación de una red de vínculos e interacciones que se diluyen y se refuerzan por medios que cambian constantemente. 
Bajo esta premisa, las uniones que le dan sentido a las prácticas sociales se definen por mutaciones de los patrones de conducta y de producción económica cada vez más complejos.

Asimismo, hay que reconocer que los procesos culturales se encuentran anclados a un territorio, tal y como lo hace Felipe González en su recorrido geográfico y simbólico del espacio metropolitano, mismo que supone la asignación e interpretación de significados para la explicación de los vínculos sociales. El autor pretende exponer cómo las prácticas sociales de la vida rural y tradicional existen y se traslapan en la experiencia de construcción de la megalópolis por medio de procesos de integración y fragmentación simultánea.

Sin embargo, a diferencia de Felipe González, considero que no solamente a través del crecimiento urbano se transforman las creencias y prácticas culturales; ejemplo de ello son los cambios en comunidades tradicionales como producto de la migración trasnacional o la interacción rural-urbana.

El indígena habita no sólo en el ámbito rural, como lo muestran los datos expuestos por Felipe González en el segundo capítulo; un gran número de ellos reside en contextos urbanos y, más aún, el ámbito de la ciudad se ha convertido en un polo de atracción a las redes de migrantes indígenas. Algunos municipios metropolitanos del Estado de México son reflejo de dicha migración.

El recorrido por las diferentes interpretaciones de la vida social que propone Felipe González nos lleva a resaltar las condiciones de distancia e integración colectiva, atravesando los vínculos cambiantes en donde prevalece el individualismo en apegos diluidos y relaciones sociales desconcentradas y ampliadas a panoramas donde la proximidad social se expresa en la paradoja de la distancia entre los individuos y la reducción del sujeto así como en el marco de la sustitución de apegos morales por reglas civiles.

El crecimiento urbano, visto desde esta óptica, da cuenta del proceso de absorción de los ámbitos rurales a una dinámica de la megalópoli, la cual está definida como la interacción de las metrópolis del valle de México y del valle de Toluca, recurriendo al estudio de las localidades que se quedaron en medio, mezcladas y entretejidas, en transición permanente hacia la indeterminación rururbana.

Es pertinente reconocer que una de las limitaciones de la mayoría de los enfoques para el análisis regional-metropolitano es la pretensión de asignar a las unidades territoriales una cierta homogeneidad (algunas localidades de los municipios llamados urbanos se pueden considerar de características rurales). En este sentido, Felipe González reconoce que los procesos culturales se mezclan en prácticas y rituales. 
Vale la pena resaltar que una característica del libro es la secuencia ordenada y el rigor científico con que se van planteando los temas. Para alcanzar este objetivo, el documento se guía por tres ejes de análisis:

1. La fragmentación y la diferenciación social que genera el proceso de aglomeración urbana.

2. La transformación de las formas territoriales de los pueblos indígenas, como efecto de la aglomeración urbana.

3. El cambio de las formas simbólicas desplegadas en el ritual y el performance.

Para Felipe González, el ritual es un acto simbólico y en sociedades fragmentadas resulta útil usar el concepto de performance, ya que a través de la liminalidad se obtienen lazos integradores, los cuales pueden ser permanentes o momentáneos, y en los que no es indispensable compartir significados. El acto simbólico pensado en estos términos genera congruencia en interpretaciones múltiples.

Haciendo una recapitulación del itinerario del autor que nos ocupa, hay que resaltar que el performance al que hace referencia está conferido al investigador en su faceta de viajero, de observador e interpretador de la experiencia humana, y se expresa por medio de los diferentes significados que se asignan a la condición social en la megalópoli. Por ejemplo, en el caso de Huixquilucan los vínculos sociales se manifiestan a través de las fiestas patronales, experiencia en la que la reciprocidad es la constante y expresa una conexión estructural entre las localidades.

Por otro lado, en el caso de Xochicuautla se muestran las características principales de los pueblos en transición, marcados por la modernidad, distinguidos por los significados de la danza y el poder referido a los vínculos de los pobladores con los representantes políticos del gobierno. En la danza de la fiesta patronal se refleja una sociedad que transita de las prácticas religiosas a las seculares por medio de los peligros que, en otras épocas, enfrentaron los arrieros al viajar a la ciudad, así como el riesgo, las diferencias de clase y de funcionalidad en la estructura social.

Cuando el autor aborda la localidad de Huitzizilapan encuentra que el componente migratorio le brinda a la comunidad nuevas características como la distribución de los asentamientos poblacionales y la unidad por el carnaval. Los huihuinches, portavoces de la tradición, personifican los vínculos sociales arraigados en la historia y multiplican los rostros, construyendo un espacio en el que cabe toda la comunidad a través del trabajo, la religión y la imaginación.

Cuando Felipe González traza su trayectoria hacia el Valle de Toluca, particularmente a Xonacatlán y Otzolotepec, dice textualmente que: 
La hipótesis del trabajo dice que a mayor metropolización (densidad demográfica, equipamiento urbanos y movilidad espacial intensa) existe una mayor diversidad de significados y valores culturales, asignados a sus prácticas, por parte de sus habitantes. En este sentido, existe una relación positiva entre metropolización y diversidad cultural (González, 2012: 190).

Una afirmación de esta naturaleza invita a la polémica, ya que implica varios desafíos importantes, en primer lugar, asigna un paralelismo a dos características esenciales de la vida moderna occidental: la metropolización y la diversidad cultural, pero ¿̇no resulta lógico que vayan de la mano? Ambas se complementan en la lógica de la racionalidad capitalista para ampliar el rango de acción de un mercado diversificado.

Por otro lado, tal y como se mencionó anteriormente, la diversificación de los significados culturales puede darse en ausencia de las características de la metropolización. Aunque hay que reconocer que este proceso escapa a los objetivos del libro de Felipe González, es justo indicar que se queda pendiente este debate.

Otro factor interesante en el trayecto que relata el texto es la importancia de las expresiones juveniles ancladas en la región, particularmente del movimiento punk. Este factor involucra un ámbito en el que Felipe González no profundiza demasiado: la transformación de las expresiones culturales como un proceso generacional.

Hay que señalar que a pesar de que prevalece una precisión geográfica en el texto, hay un punto en el que el autor reiteradamente se refiere a San Mateo Oxtotilpan como la localidad analizada en el municipio de Toluca, sin embargo, el nombre correcto de la localidad es San Mateo Oxtotitlán.

Este libro nos da cuenta de la forma en que la metrópoli transformó el modo de vida de la gente, la materialización del mito del progreso y sus contradicciones con las costumbres ancestrales. Asimismo, nos invita a reflexionar sobre el proceso en el que la ciudad crece y absorbe a las comunidades rurales, pero contra muchos pronósticos, las prácticas tradicionales no desaparecen, se adaptan a la modernidad y se vuelven expresiones híbridas de la existencia humana.

Al centrarse el estudio en el concepto de Megalópoli, se comparte la idea de que existen muchos centros urbanos, así como múltiples conexiones de tránsito y de comunicación, por lo que el cambio en el ritual se convierte en el sello de los tiempos.

Finalmente, hay que tomar en cuenta que el performance deja de ser un ritual y se traduce en un sello político de las autoridades locales, expresión de la conexión entre cultura y poder. Del mismo modo, los cambios que enfrentan las comunidades tradicionales vienen desde las entrañas de su estructura, ya que el cambio es una forma de resistir los embates de la 
modernidad y garantizar la supervivencia de la tradición. En este sentido, la aportación de Felipe González ejemplifica la transformación de la cultura, asignándole una dinámica acelerada, pero que coexiste con la tradición y las expresiones identitarias heredadas a través de la construcción de la comunidad.

Recibida: 4 de marzo de 2013. Aceptada: 13 de junio de 2013.

José JaVier Niño-Martínez Universidad Autónoma del Estado de México Correo-e: jjninom@uaemex.mx

José Javier Niño-Martínez. Es doctor en ciencia social con especialidad en sociología por El Colegio de México. Actualmente se desempeña como profesor de tiempo completo en la Facultad de Ciencias Políticas y Sociales de la Universidad Autónoma del Estado de México. Sus líneas de investigación son teoría social, estudios del desarrollo, el bienestar y la política en México. Entre sus publicaciones más recientes destacan: "Alternancia partidista e ingreso: un modelo de transición incierta" en Nelson Arteaga Botello, Juan Carlos Patiño e Ivett Tinoco García (coords.) Ciudadanía, participación politica y procesos electorales en el Estado de México, Universidad Autónoma del Estado de México, Toluca, pp. 215-240 (2011); "Las Políticas Distributivas, agendas pendientes en México y Bolivia" en Julio Aibar y Daniel Vázquez (coords.) ¿Autoritarismo o democracia? Hugo Chávez y Evo Morales, Flacso-México, México, pp. 311-315 (2009). 